CORRECTION

https://doi.org/10.1038/s41586-019-1207-2

\title{
Publisher Correction: Viral and metazoan poxins are cGAMP-specific nucleases that restrict cGAS-STING signalling
}

James B. Eaglesham, Youdong Pan, Thomas S. Kupper

\& Philip J. Kranzusch

Correction to: Nature https://www.nature.com/articles/s41586-0190928-6, published online 06 February 2019.

In this Letter, Supplementary Fig. 1 depicting gel source data was missing (the link instead downloaded another copy of Supplementary Table 1). In addition, it should be cited in the legend to Extended Data Fig. 4b: "Gel source data are available in Supplementary Fig. 1.". The link to Supplementary Table 1 providing sample source acknowledgements downloaded an incorrect table (see the Supplementary Information to this Amendment). These errors have been corrected online.

Supplementary information is available in the online version of this Amendment. 